\title{
ILES AND LES OF COMPLEX ENGINEERING TURBULENT FLOWS
}

\author{
C. Fureby ${ }^{\dagger, \ddagger}$ \\ ${ }^{\dagger}$ Div. of Weapons \& Protection, Warheads \& Propulsion \\ The Swedish Defense Research Agency, FOI, SE 14725 Tumba, Sweden \\ ${ }^{\ddagger}$ Dept. of Shipping and Marine Technology \\ Chalmers University of Technology, SE 41296 Göteborg, Sweden
}

Key words: LES, ILES, engineering applications, incompressible flow, compressible flow, flow noise, turbulent premixed combustion, cavitation.

\begin{abstract}
The present study concerns the application of Large Eddy Simulation (LES) and Implicit LES (ILES) to engineering flow problems. Such applications are often very complicated, involving both complex geometries and complex physics, such as turbulence, chemical reactions, phase changes and compressibility. The aim of the study is to illustrate what problems occur when attempting to perform such engineering flow calculations using LES and ILES, and put these in relation to the issues originally motivating the calculations. The issues of subgrid modeling are discussed with particular emphasis on the complex physics that needs to be incorporated into the LES models. Results from representative calculations, involving incompressible flows around complex geometries, aerodynamic noise, compressible flows, combustion and cavitation, are presented, discussed and compared with experimental data whenever possible.
\end{abstract}

\section{INTRODUCTION AND BACKGROUND}

A grand challenge for Computational Fluid Dynamics (CFD) is the prediction of turbulent flows. In practice, such flows often include additional physics such as compressibility, phase changes, and chemical reactions. Applications include external flows around cars, trains, ships, buildings, aircrafts and internal flows in pipes, buildings, electronic devices, mixers, food manufacturing equipment, engines, furnaces, boilers, and supersonic flows around air- and spacecrafts, missiles and in aerospace engine applications such as turbojet engines, ram and scramjets, as well as solid rocket motors. For such flows it is unlikely that we will ever have a really-deterministic predictive framework based on CFD, due to the inherent difficulty in modeling and validating all relevant sub-processes and acquiring the necessary boundary condition data. On the other hand, such cases are representative of fundamental ones for which whole-domain scalable laboratory studies are extremely difficult, and for which it is crucial to develop predictability, as well as establishing effective approaches to post-processing the simulation database.

The modeling challenge is to develop models that, although not explicitly incorporating all eddy scales, give accurate and reliable predictions for at least the large energy-containing scales. This requires the governing Navier-Stokes Equations (NSE) to be truncated in a way that the energy spectra is consistent with the $|\mathbf{k}|^{-5 / 3}$ law of Kolmogorov, with a smooth transition at the highwave-number cut-off end. The models must be designed as to minimize the contamination of the resolved part of the energy spectrum and to modify the dissipation-rate in regions where viscous 
effects are important. For flows of engineering interest the Reynolds (Re) number is often high, placing the cut-off wavenumber in the lower part of the inertial subrange. This, together with the complication of walls and additional physics, raises the demands for the modeling, incorporating also anisotropic features and multi-physics processes and considerations.

The computational challenge is to solve the model equations accurately, typically requiring a large number of time steps and grid cells. For flows in and around complex geometries, finite volume methods are superior to finite difference or element methods since they are based on the integral formulation of the governing equations and are thus conservative by construction. However, for unstructured grids efficient algorithms of higher spatial order than two are arduous to develop and implement, and unless we follow that route we cannot expect to be able to discriminate completely between the leading order truncation error and the subgrid model. Alternatively, we may instead design the overall algorithm, e.g. by means of the modified equations approach, to use the leading order truncation error as an implicit subgrid model. An additional challenge is to design grids of sufficient fidelity to resolve detailed flow features including e.g. highly curved surfaces, small-scale details, such as struts and fins, and elements of relative motion. In some cases, models may be developed that can emulate the desired features, e.g. an actuator disc representing a ship propeller, but this is not the ideal situation. Moreover, to be of use in design and concepts evaluation, the computations must be complete within a few days of building the computational grid representing the geometry and the flow domain.

Despite the steady advance in computing power and computational technology, attempts at Direct Numerical Simulation (DNS) of the NSE have been limited to simple geometries and low $\mathrm{Re},[1]$. The most widespread alternative to DNS is Reynolds Averaged Navier-Stokes (RANS) models, [2], based on a statistical treatment of the fluctuations about a stationary or slowly varying flow, and turbulence models for the mean influence of the fluctuations on the mean flow, [3]. The limitations of RANS were soon realized, and during the 1980's the concept of Large Eddy Simulation (LES) was introduced, [4-7]. In LES, all scales larger than the grid are resolved using a space-time accurate scheme, and only the effects of the small, unresolved eddy scales are to be modeled. The direct computation of the large energy containing eddies (being geometry and flow dependent) gives LES more generality than RANS but at much higher computational costs. In an attempt to develop cheaper but still accurate models, the hybrid RANS-LES was born. Detached Eddy Simulations (DES), [8], is a hybrid RANS-LES in that the boundary layer (populated with attached eddies) is assigned to RANS, and only separated regions (detached eddies) are assigned to LES. RANS, DES and LES are compared in figure 1 for the flow around a circular cylinder at $\operatorname{Re}=3900$ and 140,000, [9-12], and for the flow over a surface mounted 3D hill at $\operatorname{Re}=130,000$, [13-14]. In conclusion we find that LES is more accurate than RANS and DES, and that DES is sensitive to the inflow conditions for the turbulence variable $\tilde{v}-$ with high values of resulting in RANS-like results and low values resulting in LES-like results. In addition, we often find that the influence of the LES subgrid model is comparatively small, e.g. [14], whereas the importance of the turbulence model in RANS can be significant, [14]. When other processes are involved or when the unsteadiness of the flow is of importance LES is necessary to avoid excessive and complicated tailor-made modeling of the intrinsic coupling processes, [15], usually resulting in rather poor predictions, e.g. [16], usually with high case sensitivity. 

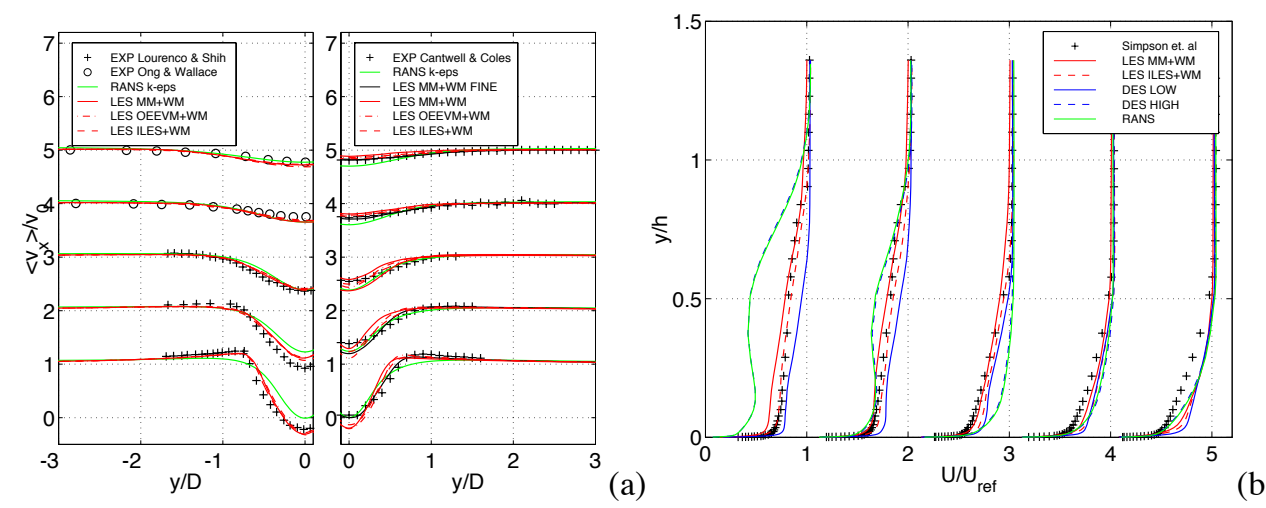

Figure 1. Comparison between RANS, DES and LES predictions for the flows past (a) a circular cylinder at $R e=3900$ (left) and $R e=140,000$ (right), and (b) the surface mounted bump at $R e=130,000$.

In the light of the above discussion, the aim of this study is to discuss the use of LES and Implicit LES (ILES), [17, 18], for engineering flow problems, including geometrical complexity and physical complexity in terms of additional physics, i.e. compressibility, chemical reactions and phase changes. To the largest extent possible we compare with experimental data and with other results. The goal is to demonstrate that realistic engineering flow problems in a variety of application areas can be studied with LES and ILES, resulting in improved predictions compared to RANS, in comparison with reference data, with manageable run-times.

\section{INCOMPRESSIBLE TURBULENT FLOW}

Incompressible flows, i.e. flows without appreciable density variations, are common in engineering applications and include low-speed aerodynamics and hydrodynamics. To demonstrate the issues of complex geometry flows we consider the flow past a submarine in model scale. Besides the conventional ship hydrodynamic design parameters, such as viscous and pressure drag, critical issues in submarine hydrodynamics include the unsteady flow over the stern part of the hull into the propeller, which is important for the propeller design, flow noise resulting from the unsteady vortical flow around the hull, the unsteady propeller flow itself and the wake. This means that we need also to include the propeller in the simulations, which is a grand challenge for unsteady RANS, and a formidable challenge for LES and DES.

We here consider the DARPA AFF-8 Suboff configuration, [19-21], figure 2, that consists of hull, sail and stern appendages, with an overall length of $\mathrm{L}=4.36 \mathrm{~m}$ and a diameter of $\mathrm{D}=0.51$ $\mathrm{m}$. The sail is located on the cylindrical hull at the top dead centre, having its leading edge at $\mathrm{x}=$ $0.92 \mathrm{~m}$ and its trailing edge at $\mathrm{x}=1.29 \mathrm{~m}$. A cap of elliptical cross-section is attached to the sail at a height of $\mathrm{h}=0.46 \mathrm{~m}$ and the stern appendages consist of four NACA 0020 profiles, defined with the trailing edge at $\mathrm{x}=4.00 \mathrm{~m}$. Experimental data is provided by the David Taylor Model Basin (DTMB) at a Re number of $\operatorname{Re}=12 \cdot 10^{6}$. The computational domain consists of the submarine hull mounted in a large cylinder and the domain extends one hull length upstream of the model and two hull lengths downstream of the model being $4 \mathrm{~L}$ in overall length and consists of about 7 million cells. For the hull an O-O topology is used, whilst for the sail and stern appendages C-O 
topologies are used and care is taken to ensure that the computational grid is appropriate for the subgrid wall model, [22], used to alleviate the computational requirements of wall resolved LES. About 10 cells are within the boundary layer on the parallel midsection of the hull resulting in $\mathrm{y}^{+} \approx 15$. Conventional open inflow and outflow conditions, [15], are used together with far-field conditions at the outer boundary and no-slip conditions on the hull.

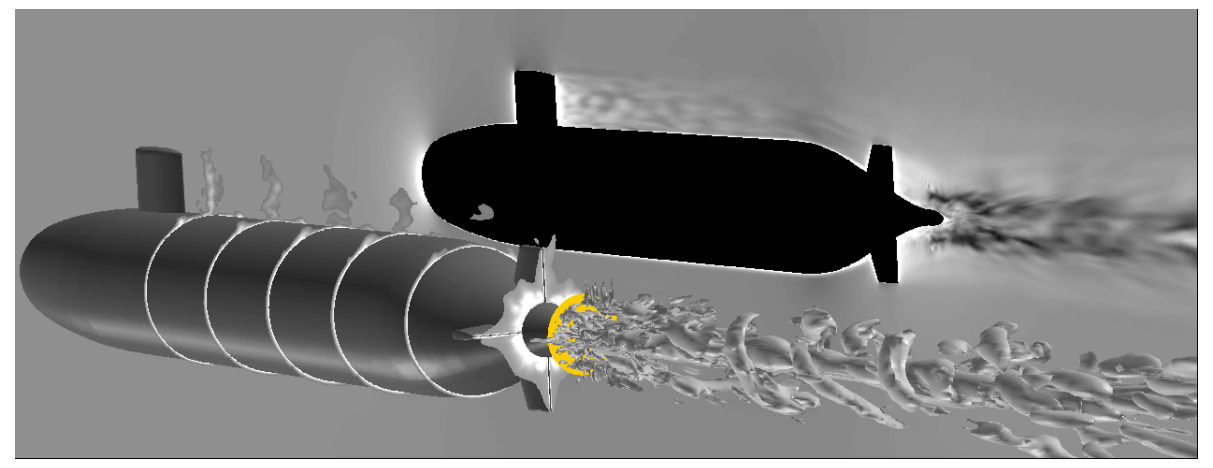

Figure 2. Submarine hydrodynamics. Schematic of the DARPA AFF-8 Suboff hull geometry, [1920], and the instantaneous flow field for a self-propelled submarine model.

The computational model typically consists of the incompressible NSE for a linear viscous fluid, and following, [23], the finite volume discretization of the low-pass filtered incompressible NSE and/or the raw incompressible NSE results in the modified equations,

$\partial_{\mathrm{t}}(\overline{\mathbf{v}})+\nabla \cdot(\overline{\mathbf{v}} \otimes \overline{\mathbf{v}})=-\nabla \overline{\mathrm{p}}+\nabla \cdot \overline{\mathbf{S}}+\boldsymbol{\tau}-\mathbf{m}^{\mathrm{v}}, \quad \nabla \cdot \overline{\mathbf{v}}=0$,

where $\mathbf{v}$ is the velocity, $\mathrm{p}$ the pressure, $\mathbf{S}=2 v \mathbf{D}$ the viscous stress tensor, $\mathbf{m}^{\mathrm{v}}=\overline{\nabla \cdot(\mathbf{v} \otimes \mathbf{v})}-\nabla \cdot(\overline{\mathbf{v}} \otimes \overline{\mathbf{v}})=$ $\overline{\nabla \cdot(\mathbf{v} \otimes \mathbf{v})}-\nabla \cdot \overline{(\mathbf{v} \otimes \mathbf{v})}+\nabla \cdot \mathbf{B}$, the unresolved transport term, $\mathbf{B}=\overline{\mathbf{v} \otimes \mathbf{v}}-\overline{\mathbf{v}} \otimes \overline{\mathbf{v}}$ the subgrid stress tensor and $\tau$ the truncation error associated with the numerical algorithm. The unresolved transport term is usually simplified such that only the subgrid force vector $\nabla \cdot \mathbf{B}$ is retained. Moreover, the subgrid stress tensor is commonly modeled using an explicit subgrid model such as:

- the subgrid-viscosity model (VM), $\mathbf{B}=-2 v_{\mathrm{k}} \overline{\mathbf{D}},[6]$,

- the mixed model (MM), $\mathbf{B}=\overline{\overline{\mathbf{v}} \otimes \overline{\mathbf{v}}}-\overline{\overline{\mathbf{v}}} \otimes \overline{\overline{\mathbf{v}}}-2 v_{\mathrm{k}} \overline{\mathbf{D}},[6]$.

In contrast to explict LES, where high-order central schemes are used to discretize both the convective and diffusive terms, such that $\boldsymbol{\tau}=\boldsymbol{\tau}_{\mathrm{CD}}$, implicit LES is based on high-order non-oscillatory schemes for the convective terms, such that $\boldsymbol{\tau}=\boldsymbol{\tau}_{\mathrm{CD}}+\boldsymbol{\tau}_{\mathrm{NO}}$, where $\boldsymbol{\tau}_{\mathrm{NO}}$ is associated with the flux limiter $\Psi$ and the upwind-biased component of the high-order non-oscillatory scheme. Comparing modified equations from LES and ILES implies that $\boldsymbol{\tau}_{\mathrm{NO}}$ may be interpreted as an implicit subgrid model such that $\mathbf{B}=\boldsymbol{\tau}_{\mathrm{NO}} \approx \mathbf{C}(\nabla \overline{\mathbf{v}})^{\mathrm{T}}+(\nabla \overline{\mathbf{v}}) \mathbf{C}^{\mathrm{T}}$, [23], where $\mathbf{C}=\chi(\overline{\mathbf{v}} \otimes \mathbf{d})$ defines the tensor-valued subgrid viscosity with $\chi=\frac{1}{2}(1-\Psi)\left(\beta^{-}-\beta^{+}\right)$and $\beta^{ \pm}=\frac{1}{2}(\operatorname{sign}(\overline{\mathbf{v}}) \pm 1)$.

For VM and MM we use the One Equation Eddy Viscosity Model, [24], with coefficients evaluated under the assumption of an infinite inertial sub-range. For ILES we use the Flux Corrected Transport (FCT) algorithm, [15]. The discretized equations are merged to form a Poisson 
equation for the pressure. The scalar equations are solved sequentially, with iteration over the coupling terms and the pressure-velocity coupling is handled with a PISO-type procedure. To emulate the propeller, an actuator-disc model is used in which a source term is added to the momentum equation in the region of the propeller that accelerates the flow giving thrust and a rotating slipstream. The source term is evaluated using a lifting-line technique, [25], using Goldsteins kappa-theory, [26], to account for the number of blades.

The main flow features are shown in figure 2 . The flow past the forebody is dominated by the stagnation point region and the favorable pressure gradient on the foreshoulder that gradually develop into a fully turbulent boundary layer. The flow over the midbody is dominated by the horseshoe vortex, originating from the roll-up of the boundary layer just ahead of the sail, and by the sail-wake. The horseshoe-vortex continues along the sail forming additional vortices towards the trailing edge of the sail that interact with the hull-boundary layer and the horseshoe-vortex, forming an unsteady wake behind the sail. As the horseshoe-vortex is transported further aft, it loses its coherence and flattens towards the hull. During this process it interacts with the hull-boundary layer, developing a complex near-wall flow with embedded vortices approaching the tapered stern. Such complex flow pattern on the sail-side of the hull creates transport of momentum across the hull, which affects the velocity and shear stress distribution in the hull-boundary layer. The sail-tip vortex pair persists far downstream but does not interact with other vortices and passes well above the propeller. The flow over the sail-cap is dominated by the flow turning, and the development of the sail-tip vortex pair at the junction between the sail and sail-cap. Towards the stern, vortex sheets are formed along the rudders and counter-rotating tip vortices and horseshoe vortices are formed on each of the rudders. These vortices becomes embedded in the thick boundary layer that is affected by the tapering of the hull and the adverse pressure gradient. Redistribution of Reynolds stresses are also found to take place in the stern due to the cross flow, caused by the unsteady sail wake and horseshoe-vortex. As can be seen in figure 3 the presence of the propeller affects the flow over the stern. This is caused by the pressure distribution due to the propeller-induced suction. Only just upstream of the propeller we note the influence of the tangential and radial velocity distributions caused by the rotating propeller. Downstream of the propeller the flow is different and primarily swirl dominated.
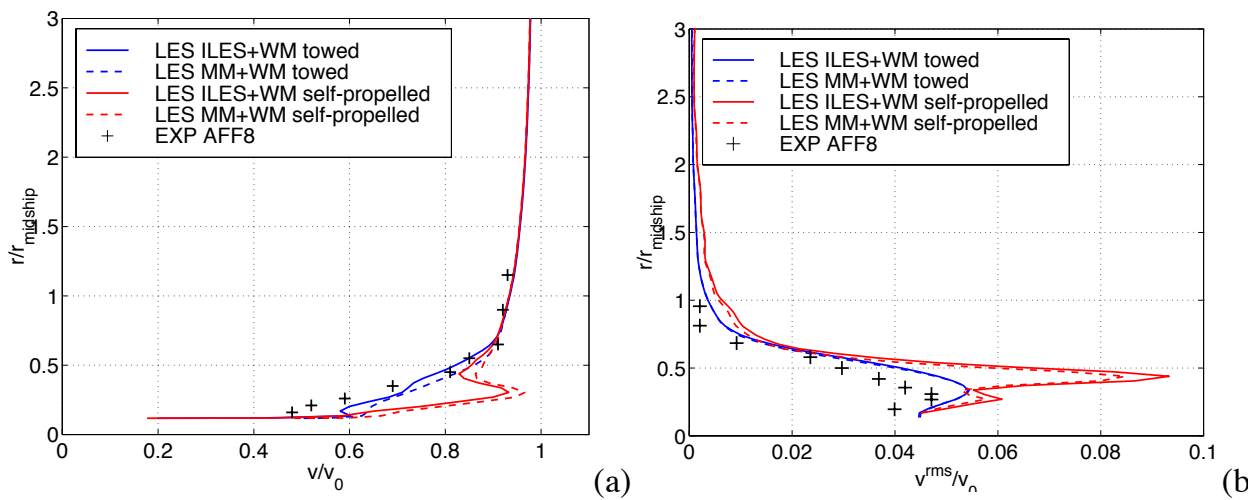

Figure 3. Submarine hydrodynamics. Comparison of predicted and measured (a) time and azimuthally averaged velocity $\left\langle\overline{\mathrm{v}}_{1}\right\rangle_{\varphi} / \mathrm{v}_{0}$, and (b) velocity fluctuations $\left\langle\mathrm{v}_{\mathrm{x}}^{\mathrm{rms}}\right\rangle_{\varphi} / \mathrm{v}_{0}$ at $\mathrm{x} / \mathrm{L}=0.978$. 
Figure 3 a presents a comparison of the time and azimuthally averaged streamwise velocity component, $\left\langle\overline{\mathrm{v}}_{1}\right\rangle_{\varphi} / \mathrm{v}_{0}$, between LES and measurement data for the towed and self-propelled hulls, at $\mathrm{x} / \mathrm{L}=0.978$. Good agreement between LES predictions and data is obtained, whereas further away from the hull $\left\langle\overline{\mathrm{v}}_{1}\right\rangle_{\varphi}$ approaches $\mathrm{v}_{0}$ asymptotically. In the self-propelled case, the slipstream clearly is visible, revealing that the propeller entrains fluid mainly from the hull-boundary layer, which in turn affects the levels of fluctuations in the boundary layer. Figure $3 \mathrm{~b}$ presents a comparison of the streamwise rms-velocity fluctuations $\left\langle v_{x}^{r m s}\right\rangle_{\varphi} / v_{0}$. Again, the influence of the explicit or implicit subgrid model is comparatively small. As compared to the towed hull case the self-propelled hull case produces a bi-modal rms-velocity fluctuation profile, with the outer peak associated with the radius of the propeller and the inner peak value associated with the peak in the mean velocity profile, associated with the boundary layer flow.

\section{AERODYNAMIC NOISE}

Flow noise has become a more significant contributor to the interior noise level in cars and highspeed trains at high cruising speeds due to developments in noise reduction from the engine and the tires and wheels, respectively. In this context, the flow noise consists of aspiration noise and aerodynamic noise. The aspiration noise has been reduced greatly by implementing novel sealing techniques, whereas the aerodynamic noise, generated by the high Re-number turbulent external flow, remains a problem to be solved by experimental and computational approaches. The noise characteristics are found to be very sensitive to design details, including door handles, slits, Apillars, wing mirrors etc. since such details usually creates additional flow unsteadiness. This is computationally challenging since the simulations need not only to include the complete, often rather complicated geometry, but also the unsteady effects which are responsible for the pressure (or density) fluctuations recognized as noise. More precisely, flow noise consists of sequence of compressions and rarefactions, traveling with the speed of sound, c, of the fluid.

If the compressible NSE is used as a computational model for the aerodynamic noise, this model needs to properly resolve the small density variations of the longitudinal waves associated with the aerodynamic noise. This puts hard requirements on the accuracy of the scheme in space and time, requiring low dissipation as well as fully non-reflective boundary conditions. Other methods include perturbation techniques, e.g. [27], which reduce the overall computational time by solving separately the incompressible and acoustic parts by using two different grids and schemes. Methods based on acoustic analogy theory, [28], also allow one to decompose the problem into two parts: prediction of the sources and subsequent propagation by using FfowcsWilliams Hawkings equation, [29]. The advantage is that Ffowcs-Williams Hawkings equation is an explicit equation that does not require iterative solvers. Kirchhoff's theory, [30], also leads to a two stage method, but sound sources have to be placed and computed on a virtual surface around the vehicle beyond which non-linear effects are negligible. An advantage of this method with respect to the previous one is that only surface integrals need to be evaluated whereas the Ffowcs-Williams Hawkings equation involves both surface and volume integrals. For the above reasons, most flow noise problems are solved using the Ffowcs-Williams Hawkings equation in conjunction with the incompressible NSE. In this work, ILES and LES will be used to predict the aerodynamic sources, so that the full model reads, 
$\partial_{\mathrm{t}}(\overline{\mathbf{v}})+\nabla \cdot(\overline{\mathbf{v}} \otimes \overline{\mathbf{v}})=-\nabla \overline{\mathrm{p}}+\nabla \cdot \overline{\mathbf{S}}+\boldsymbol{\tau}-\mathbf{m}^{\mathrm{v}}, \quad \nabla \cdot \overline{\mathbf{v}}=0$,

$\mathrm{p}^{\prime}=\frac{\rho_{0}}{4 \pi} \int_{\mathrm{V}}\left[\frac{1}{\mathrm{c}^{2}|\mathbf{x}|}(\mathbf{l} \otimes \mathbf{l}) \cdot \ddot{\overline{\mathbf{T}}}+\frac{3}{\mathrm{c}^{2}|\mathbf{|}|^{2}}(\mathbf{l} \otimes \mathbf{I}-\mathbf{I}) \cdot \dot{\overline{\mathbf{T}}}+\frac{3}{\mathrm{c}^{2}|\mathbf{x}|^{3}}(\mathbf{l} \otimes \mathbf{I}-\mathbf{I}) \cdot \overline{\mathbf{T}}\right] \mathrm{dV}+\frac{\rho_{0}}{4 \pi} \int_{\mathrm{S}}\left[\frac{1}{\mathrm{cr}}(\dot{\overline{\mathrm{p}}} \mathbf{l} \cdot \mathbf{n}-\dot{\overline{\mathbf{S}}} \mathbf{l} \cdot \mathbf{n})+\frac{1}{|\mathbf{x}|^{2}}(\overline{\mathrm{p}} \mathbf{l} \cdot \mathbf{n}-\overline{\mathbf{S}} \mathbf{l} \cdot \mathbf{n})\right] \mathrm{d} S$,

where $\overline{\mathbf{T}}=\rho \overline{\mathbf{v}} \otimes \overline{\mathbf{v}}-\overline{\mathbf{S}}+\left(\overline{\mathrm{p}}-\mathrm{c}^{2} \rho\right) \mathbf{I}$ is the Lighthill aerodynamic source tensor, $\mathbf{n}$ the outward pointing unit normal vector to the surface $S, \mathbf{l}$ the unit normal vector pointing from the source to the observer, $\rho \approx \rho_{0}$ whereas the dots denote time derivatives. The volume integral, i.e. the quadrupole term, is usually negligible in comparison to the surface integral, i.e. the dipole term, for low Manumber flows. The derivation of this model can be found in [28].

In order to illustrate the problem of predicting flow noise we here consider the flow around a generic wing mirror and the resulting flow noise at Re $\approx 7 \cdot 10^{5}$. Höld et al, [31], and Siegert et al, [32], reported detailed experimental measurements of the acoustic fields in the vicinity of a wing mirror mounted on a plate in an aeroacoustic wind tunnel. The wing mirror is composed of a half cylinder $0.20 \mathrm{~m}$ in diameter and length topped by a quarter of a sphere of the same diameter. It is mounted on a flat plate $1.60 \mathrm{~m}$ wide, $2.40 \mathrm{~m}$ long and $0.05 \mathrm{~m}$ thick. The wing mirror is mounted $0.90 \mathrm{~m}$ downstream of the elliptically shaped leading edge. The computational model replicates the experimental geometry with the far field boundaries placed $1.00 \mathrm{~m}$ to the sides and above the plate. The lower computational domain coincides with the symmetry plane of the plate. Standard open inflow/outflow boundary conditions are used, whereas on the wing mirror and the plate noslip conditions are used. Two grids of 1.5 and 3.0 million cells are used.
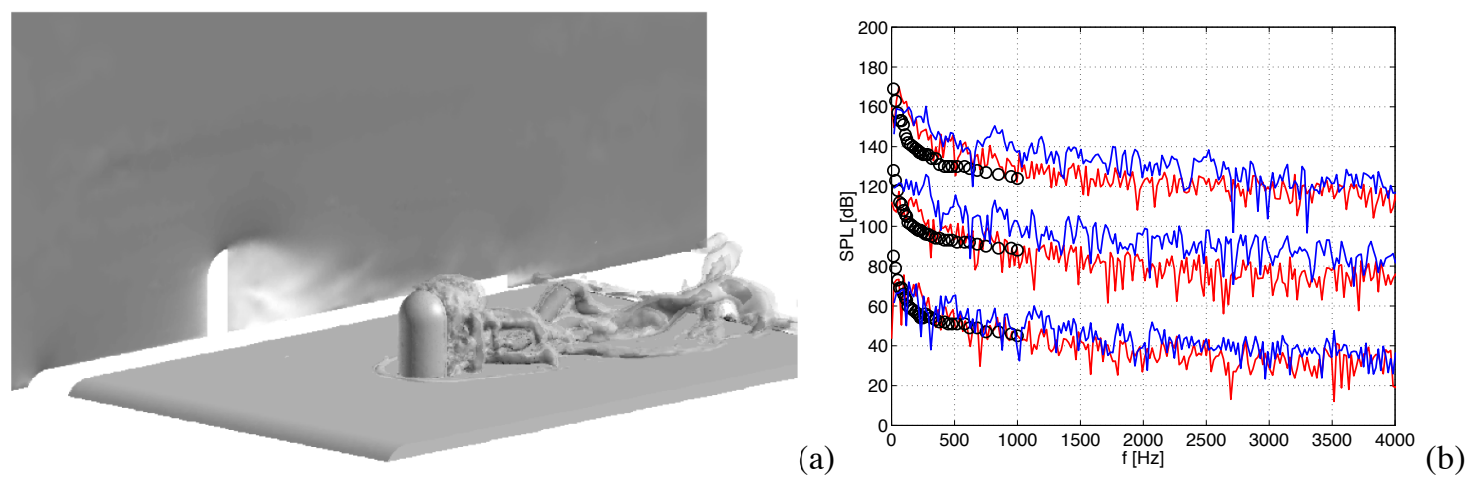

Figure 4. Aerodynamic noise. (a) Perspective view of the flow around the wing mirror, including the grid and (b) comparisons of sound pressure levels at discrete points around the wing mirror. Note that the different spectra are spaced by $40 \mathrm{~dB}$ in the vertical direction to facilitate comparison.

Figure 4a shows a perspective view of the flow around the wing mirror, from which it is evident that it creates a large complex wake with eddy structures spanning a range of scales. The flow accelerates to about $95 \mathrm{~m} / \mathrm{s}$ at the tip of the mirror, and the separation region extends over a distance about twice the mirror height. A horseshoe vortex system is observed to form at the upstream junction between the plate and the wing mirror, and to extend along the wing mirror, and further along the plate whereafter it rapidly looses coherence and gradually disappears. The wake behind the wing mirror actually hinges on an arch-shaped vortical structure observed only in the time-averaged results. This arch-shaped vortical structure is found to meander from side to side, 
and is most likely not complete at any instant. In addition, we notice a quasi-periodic vortex shedding at the vertical edges of the cylindrical part of the mirror resulting in two regions of high rms fluctuations about one diameter downstream of the mirror. Similarly, the vortical structures shed at the top of the mirror impact some two diameters downstream of the mirror. In figure $4 \mathrm{~b}$ we show comparisons of the sound pressure levels at a few points around the wing mirror. The results from the Ffowcs-William Hawking acoustic model, using the LES and the ILES data, are found to match well with the experimental measurements, [31]. However, the predicted spectra are more jagged than the experimental spectra, a feature also observed by other authors, but not fully understood. One reason may be that the experimental data is filtered whereas the simulation data is not. It appears that the ILES model somewhat overpredicts the sound pressure level in comparison to the data, whereas the LES result in a very good overall agreement. Moreover, the influence of spatial resolution appears very limited for both models.

\section{COMPRESSIBLE TURBULENT FLOWS}

In contrast to incompressible flows, compressible flows involve significant density changes, and are important in aerodynamics and in turbomachinery applications. To demonstrate the inherent challenges of compressible turbulent flows we focus on the flow around a generic projectile, and particularly on its afterbody. Behind the projectile, the pressure is lower than its ambient value, causing base drag, [33]. Early attempts to predict afterbody flows are reviewed in [34], however, solving the underlying NSE offers the ability to better predict the details of the flow, and to take out the assumptions of the simpler methods. Both early and more recent RANS calculations, [3536], fail to predict these flows, and improved models are required. This has been investigated using LES, [37], and DES, [38-39], and comparison with experimental data, [40], has shown superior agreement compared to RANS, e.g. [39]. In addition, we here focus on means for reducing the base drag by injecting fluid into the near-wake, thereby expanding the recirculation region by pushing the stagnation point further downstream, figure 5a. Experimental data for this situation, with different bleed rates, is available in Mathur \& Dutton, [41], and provides an excellent validation of compressible flow models as well as to elucidate the flow physics.

The fluid dynamic model used consists of the compressible NSE, describing conservation of mass, momentum, and energy of a Newtonian fluid obeying Fourier's law of thermal conduction and the ideal gas law. A finite volume discretization of the low-pass filtered compressible NSE and/or the raw compressible NSE results in the modified equations,

$\left\{\begin{array}{l}\partial_{\mathrm{t}}(\bar{\rho})+\nabla \cdot(\bar{\rho} \tilde{\mathbf{v}})=\tau^{\rho}-\mathrm{m}^{\rho}, \\ \partial_{\mathrm{t}}(\bar{\rho} \tilde{\mathbf{v}})+\nabla \cdot(\bar{\rho} \tilde{\mathbf{v}} \otimes \tilde{\mathbf{v}})=-\nabla \tilde{\mathbf{p}}+\nabla \cdot \tilde{\mathbf{S}}+\tau^{\mathrm{v}}-\mathbf{m}^{\mathrm{v}}, \\ \partial_{\mathrm{t}}(\bar{\rho} \tilde{\mathbf{e}})+\nabla \cdot(\bar{\rho} \tilde{\mathbf{v}} \tilde{\mathbf{e}})=\nabla \cdot((-\tilde{\mathbf{p}} \mathbf{I}+\tilde{\mathbf{S}}) \tilde{\mathbf{v}}+\tilde{\mathbf{h}})+\tau^{\mathrm{e}}-\mathrm{m}^{\mathrm{e}},\end{array}\right.$

where $\rho$ is the density, e the total energy, $\mathbf{h}$ the heat flux vector, $\tau^{\mathrm{k}}$ the truncation error associated with the numerical algorithm, with $\mathrm{k}=\{\rho, \mathrm{v}, \mathrm{e}\}$, and, 


$$
\left\{\begin{array}{l}
\mathrm{m}^{\rho}=\overline{\nabla \cdot(\rho \mathbf{v})}-\nabla \cdot(\bar{\rho} \tilde{\mathbf{v}})=\overline{\nabla \cdot(\rho \mathbf{v})}-\nabla \cdot \overline{(\rho \mathbf{v})}, \\
\mathbf{m}^{\mathrm{v}}=\overline{\nabla \cdot(\rho \mathbf{v} \otimes \mathbf{v}+\mathrm{p} \mathbf{I}-\mathbf{S})}-\nabla \cdot(\rho \tilde{\mathbf{v}} \otimes \tilde{\mathbf{v}}+\tilde{\mathrm{p}} \mathbf{I}-\tilde{\mathbf{S}})=\overline{\nabla \cdot(\rho \mathbf{v} \otimes \mathbf{v}+\mathrm{pI}-\mathbf{S})}-\nabla \cdot \overline{(\rho \mathbf{v} \otimes \mathbf{v}+\mathrm{pI}-\mathbf{S})}+\nabla \cdot \mathbf{B}, \\
\mathrm{m}^{\mathrm{e}}=\overline{\nabla \cdot((\rho \mathrm{e}+\mathrm{p}-\mathbf{S}) \mathbf{v}-\mathbf{h})}-\nabla \cdot((\bar{\rho} \tilde{\mathrm{e}}+\tilde{\mathrm{p}}-\tilde{\mathbf{S}}) \tilde{\mathbf{v}}-\tilde{\mathbf{h}})=\overline{\nabla \cdot((\rho \mathrm{e}+\mathrm{p}-\mathbf{S}) \mathbf{v}-\mathbf{h})}-\nabla \cdot \overline{((\rho \mathrm{e}+\mathrm{p}-\mathbf{S}) \mathbf{v}-\mathbf{h})}+\nabla \cdot \mathbf{b},
\end{array}\right.
$$

where $\mathbf{B}=\bar{\rho}(\overline{\mathbf{v} \otimes \mathbf{v}}-\tilde{\mathbf{v}} \otimes \tilde{\mathbf{v}})+\tilde{\mathbf{S}}-\overline{\mathbf{S}}+(\overline{\mathbf{p}}-\tilde{\mathrm{p}}) \mathbf{I}$ and $\mathbf{b}=\bar{\rho}(\overline{\mathbf{v e}}-\tilde{\mathbf{v}} \tilde{\mathbf{e}})+\tilde{\mathbf{S}} \tilde{\mathbf{v}}-\overline{\mathbf{S v}}+\overline{\mathbf{p} \mathbf{v}}-\overline{\mathrm{p}} \tilde{\mathbf{v}}+\tilde{\mathbf{h}}-\overline{\mathbf{h}}$. Evidently, the subgrid stress tensor and flux vector in the compressible case include more physics than for the incompressible case. Explicit closure models are typically borrowed from the incompressible case, and corrected for the variable density. This may be acceptable for some supersonic flows, but not for hypersonic flows that recently have received renewed attention. Here, we have chosen to use an explicit LES model utilizing the mixed model $\mathbf{B}=\bar{\rho}(\overline{\tilde{\mathbf{v}} \otimes \tilde{\mathbf{v}}}-\overline{\tilde{\mathbf{v}}} \otimes \overline{\tilde{\mathbf{v}}})-2 \mu \tilde{\mathbf{D}}{ }_{\mathrm{D}}$ and $\mathbf{b}=\bar{\rho}(\overline{\tilde{\mathbf{v}} \tilde{\mathbf{e}}}-\overline{\tilde{\mathbf{v}}} \overline{\tilde{\mathbf{e}}})-\mu / \operatorname{Pr}_{\mathrm{T}} \nabla \tilde{\mathrm{e}}$, [42], and an implicit LES model with $\mathbf{B} \approx \bar{\rho}\left(\mathbf{C}(\nabla \tilde{\mathbf{v}})^{\mathrm{T}}+(\nabla \tilde{\mathbf{v}}) \mathbf{C}^{\mathrm{T}}\right)$ and $\mathbf{b} \approx \bar{\rho} \mathbf{C} \nabla \tilde{e}$ in which $\mathbf{C}=\chi(\tilde{\mathbf{v}} \otimes \mathbf{d})$ and $\chi=\frac{1}{2}(1-\Psi)\left(\beta^{-}-\beta^{+}\right)$with $\beta^{ \pm}=\frac{1}{2}(\operatorname{sign}(\tilde{\mathbf{v}}) \pm 1)$.

The spatial discretization is performed using an unstructured second-order accurate finite volume method using polyhedral CV's for the decomposition of the computational domain and a cell-centered, co-located variable arrangement. For the explicit LES we use central differencing for both the convective and diffusive terms, whereas for the ILES we use either the classical vanLeer, [43], or FCT algorithms, [44]. These two schemes give raise to different limiters $\Psi$ and hence to different subgrid models. Finally, the time-integration used employs the two-stage total variational diminishing Runge-Kutta scheme of Gottlieb \& Shu, [45].

The experiments of Dutton et al, [40-41], are performed at Ma 2.46 for an afterbody with a diameter of $\mathrm{D}=63.5 \mathrm{~mm}$ with a bleed orifice of $\mathrm{d}=25.4 \mathrm{~mm}$. The ambient velocity, pressure and temperature are $\mathrm{v}_{\infty}=576 \mathrm{~m} / \mathrm{s}, \mathrm{p}_{\infty}=515 \mathrm{kPa}$ and $\mathrm{T}_{\infty}=294 \mathrm{~K}$, for the zero bleed case and $\mathrm{v}_{\infty}=576 \mathrm{~m} / \mathrm{s}$, $\mathrm{p}_{\infty}=471 \mathrm{kPa}$, and $\mathrm{T}_{\infty}=300 \mathrm{~K}$, for the bleed cases that are characterized by the injection parameter $\mathrm{I}=\rho_{\mathrm{j}} \mathrm{v}_{\mathrm{j}} \mathrm{d}^{2} / \rho_{\infty} \mathrm{v}_{\infty} \mathrm{D}^{2}$, where $\mathrm{v}_{\mathrm{j}}$ and $\rho_{\mathrm{j}}$ are the bleed velocity and density. The computational domain consists of a cylinder with an outer radius of 3D, extending 4D upstream of and 6D downstream of the base plane. Two grids with 2 and 4 million cells, respectively, have been used. Supersonic and subsonic inflow conditions are applied at the upstream boundary and for the bleed jet, respectively. Freeflow conditions are imposed at the downstream boundary and in the radial direction, and no-slip adiabatic boundary conditions are imposed on the afterbody.
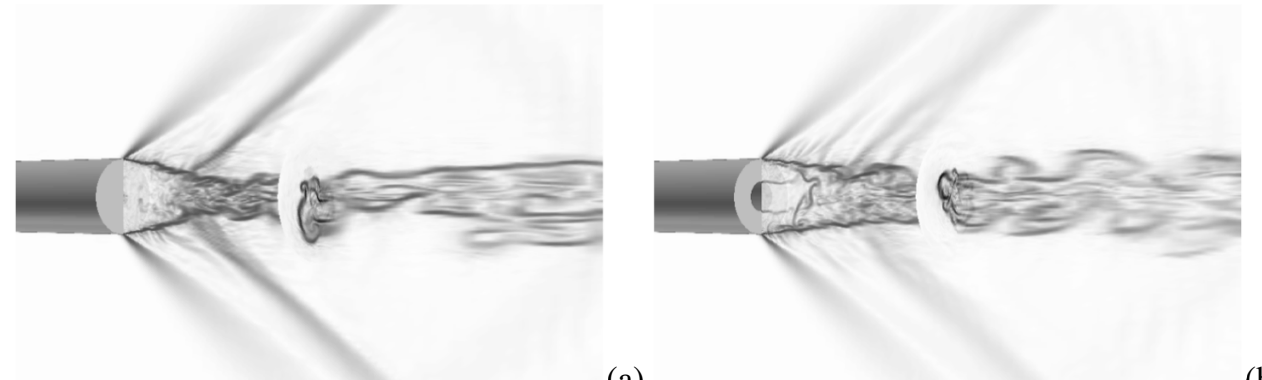

(a)

(b)

Figure 5. Supersonic base flow. Perspective view showing emulated schlieren images from the (a) zero bleed case $(I=0)$ and (b) the strongest bleed case $(I=0.0226)$ from ILES. 
In figure 5 we show numerical schlieren images at the centerplane and at an axial plane 2D downstream of the base from two cases with $\mathrm{I}=0$ and $\mathrm{I}=0.0226$. Irrespectively of the value of $\mathrm{I}$, the flows are unsteady and fully 3D. At the base corners, the expansion fans are clearly visible, followed by the near-wake recirculation bubble, the recompression waves and the trailing wake. At low bleed rates, the flow is reversed virtually out to the corners whereas at higher bleed rates the flow only becomes reversed in the annular region around the bleed jet and downstream of the bleed jet. The outer shear layer forms along the body and develops toward reattachment, whereupon it forms the far wake. The inner shear layer occurs only in the bleed cases - its strength dependent on I. The pressure gradient and the shear stress gradients in the outer shear layer decrease as the outer shear layer matures as turbulent mixing entrains ambient fluid into the wake. Base bleed widen the wake, increase the mixing, reduces the shear stresses, but by directing the bleed into the recirculation region, downstream mixing is delayed. For $\mathrm{I}=0$ the vorticity is dominated by rings (in the outer shear layer) developing into axial vortices in the region of mean reattachment showing signs of shedding. For $I>0$, the outer vortex rings are supplemented by inner vortex rings developing within the bleed jet shear layer. The vortex rings are short-lived and are only present in the near-wake, and may occasionally alternate with helicoidal vortices. In the reattachment region, large coherent structures are found in both the LES and the experimental data. Virtually no differences in global behavior can be distinguished between the LES and the ILES results, suggesting that the intrinsic differences between these models do not significantly influence the dynamics of the large (resolved) coherent structures.

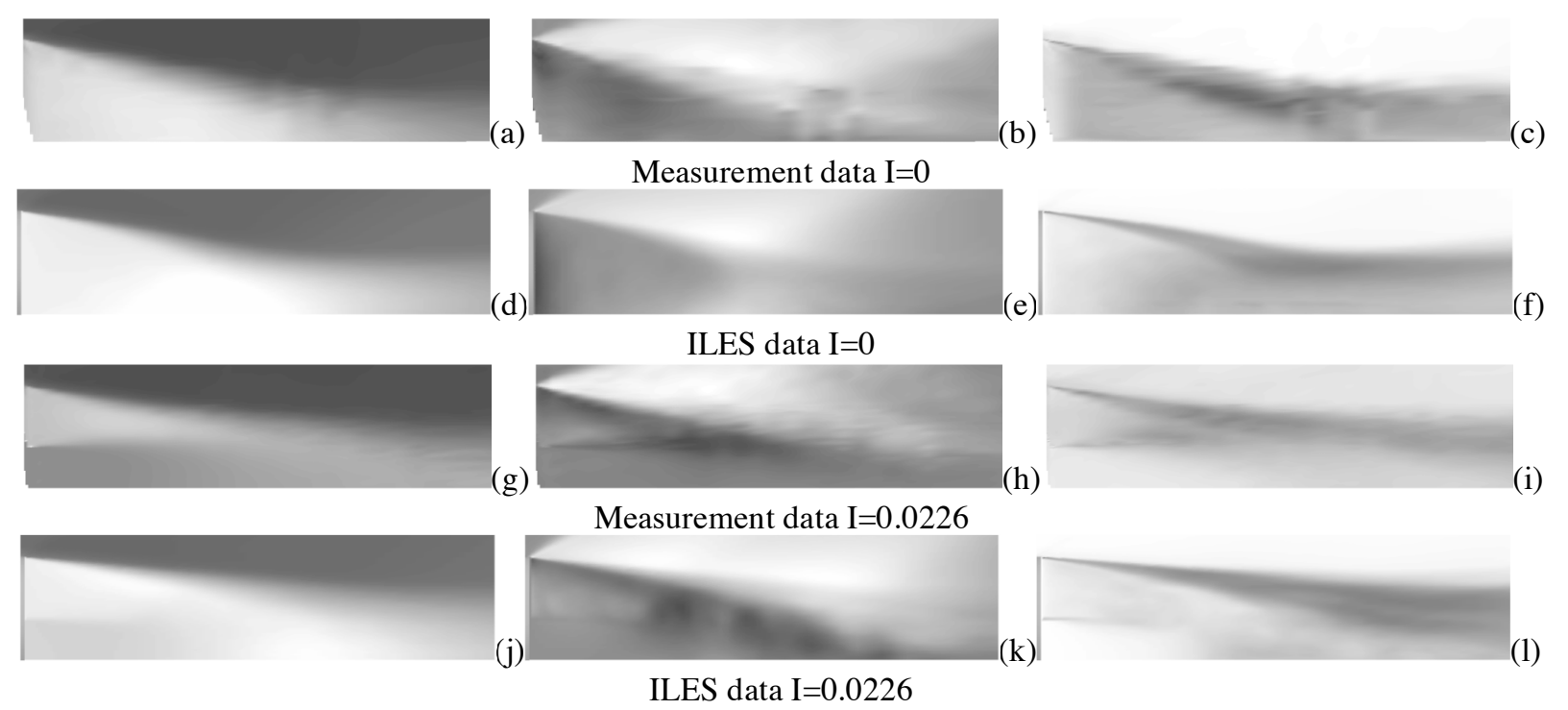

Figure 6. Supersonic base flow. Figures (a) to (f) show measurement data (top row) and ILES results (bottom row) at $\mathrm{I}=0$, respectively. Figures ( $\mathrm{g}$ ) to (l) show measurement data (top row) and ILES results (bottom row) at $\mathrm{I}=0.0226$, respectively. First column shows $\left\langle\mathrm{v}_{\mathrm{x}}\right\rangle$, second $\left\langle\mathrm{v}_{\mathrm{r}}\right\rangle$ and third $\mathrm{k}$.

The influence of the bleed rate is presented in figure 6, where contours of the time-averaged axial velocity $\langle\tilde{\mathrm{v}}\rangle_{\mathrm{x}}$, radial velocity $\langle\tilde{\mathrm{v}}\rangle_{\mathrm{r}}$ and axial rms velocity fluctuations $\mathrm{v}_{\mathrm{x}}^{\mathrm{rms}}$ at the center- 
plane for $\mathrm{I}=0$ and 0.0226 are shown. The main features of the flow, the turning of the flow over the base corner expansion, the Primary Recirculation Region (PRR) (relevant for small values of I), the bleed jet, and the Secondary Recirculation Region (SRR) between the bleed jet and the outer shear layer (relevant for large values of I), are clearly visible. Irrespectively of I, the $\langle\tilde{\mathrm{v}}\rangle_{\mathrm{r}}$ component is considerably smaller than the $\langle\tilde{\mathrm{v}}\rangle_{\mathrm{x}}$ component emphasizing the anisotropy of the flow. The freestream flow angles downstream of the base corner are consistent with data for each case [41-42], and the Prandtl-Meyer turning angle based on the estimated base pressure ratio and the freestream Ma number of 2.46. With increasing bleed flow, the size and strength of the PRR (bounded in the axial direction by the forward and rear stagnation points, respectively) decrease until it finally disappears at $\mathrm{I} \approx 0.0226$, as the bleed flow penetrates the outer shear layer reattachment region. In addition, the forward stagnation point is displaced progressively downstream of the base plane, and the radial extent of the forward portion of the PRR decreases. With increasing bleed, the SRR near the base annulus becomes more evident. Much of the reverse flow in the PRR is oriented parallel to the axis of symmetry which is in contrast to the $I=0$ case, in which the recirculating flow is directed towards the point of separation. The predicted axial and radial velocity distributions are in good agreement with data, [41-42], for both LES and ILES, although only ILES results are presented in figure 6 . The distribution of $\langle\tilde{v}\rangle_{x}$ in the free shear layer downstream of the base appears to be separated in an inner region and an outer region, of which the former appears to diverge more rapidly towards the centerline. The inner region finally overtakes the outer region and consumes most of the total shear layer width. As the outer flow approaches the axis of symmetry, the magnitude of the radial component $\langle\tilde{\mathrm{v}}\rangle_{\mathrm{r}}$ continues to increase due to the axisymmetric effect. The value of I clearly affects the strength of the recompression waves: for $\mathrm{I}=0$ a strong recompression is found in the predictions, whilst at higher values of I this weakens to finally expire at $\mathrm{I}=0.0226$. Comparing the predicted and measured $\mathrm{v}_{\mathrm{x}}^{\mathrm{rms}}$ distributions show that the simulations models can also predict the second order statistical moments with reasonable accuracy, and that the influence of the bleed jet on $\mathrm{v}_{\mathrm{x}}^{\mathrm{rms}}$ is significant.

\section{CHEMICALLY REACTIVE FLOWS}

Combustion is a ubiquitous process where the released chemical energy can be used in a controlled fashion in chemical propulsion systems, such as in air-breathing engines (turbojets, ram- and scramjets and pulse detonation engines), solid fuel rocket motors, conventional IC engines, gas turbines, as well as in furnaces. Combustion can also occur in an uncontrolled fashion such as in explosions and fires, where the released chemical energy usually causes damage to humans, constructions and to society. Combustion is also important in launch systems and more recently also in certain weapon systems. Almost all combustion of practical interest takes place in the presence of turbulence and although our understanding of turbulent combustion is constantly improving, there can be little doubt that this still represents a serious bottleneck.

The current development of combustion systems is based primarily on empirical data and simple design models that will not be adequate for multi-variate optimization of fuel, emissions and signatures or if new concepts are to be examined. Alternatives involve the use of advanced diagnostics and state-of-the-art computations. Detailed experiments are very expensive and complicated, however, recent advances in high-resolution non-intrusive diagnostics together with fast 
and reliable data reduction algorithms have enabled detailed studies of laboratory devices and efficient data collection from field measurements, [46]. The rapid advance on supercomputing and massively parallel processing has brought forth computational systems large enough to handle combustion, [47]. Computational combustion has paved new routes to perform numerical simulations to guide experimental design and to aid in parametric studies, enabling the variation of parameters and to examine issues that cannot be studied otherwise.

The equations that describe chemically reactive flows are the conservation and balance equations of mass, momentum and energy, describing the convective motion of the fluid, the diffusive transport processes and the chemical reactions. A finite volume discretization of the lowpass filtered reactive NSE and/or the raw reactive NSE results in the modified equations,

$\left\{\begin{array}{l}\partial_{\mathrm{t}}(\bar{\rho})+\nabla \cdot(\bar{\rho} \tilde{\mathbf{v}})=\tau^{\rho}-\mathrm{m}^{\rho} \\ \partial_{\mathrm{t}}\left(\bar{\rho} \tilde{\mathrm{Y}}_{\mathrm{i}}\right)+\nabla \cdot\left(\bar{\rho} \tilde{\mathbf{v}} \tilde{\mathrm{Y}}_{\mathrm{i}}\right)=\nabla \cdot \tilde{\mathbf{j}}_{\mathrm{i}}+\overline{\mathrm{W}}_{\mathrm{i}}+\tau^{\mathrm{Y}_{\mathrm{i}}}-\mathrm{m}^{\mathrm{Y}_{\mathrm{i}}} \\ \partial_{\mathrm{t}}(\bar{\rho} \tilde{\mathbf{v}})+\nabla \cdot(\bar{\rho} \tilde{\mathbf{v}} \otimes \tilde{\mathbf{v}})=-\nabla \tilde{\mathrm{p}}+\nabla \cdot \tilde{\mathbf{S}}+\tau^{\mathrm{v}}-\mathbf{m}^{\mathrm{v}} \\ \partial_{\mathrm{t}}(\bar{\rho} \tilde{\mathbf{e}})+\nabla \cdot(\bar{\rho} \tilde{\mathbf{v}} \tilde{\mathbf{e}})=\nabla \cdot((-\tilde{\mathrm{p}} \mathbf{I}+\tilde{\mathbf{S}}) \tilde{\mathbf{v}}+\tilde{\mathbf{h}})+\bar{\rho} \tilde{\sigma}+\tau^{\mathrm{e}}-\mathrm{m}^{\mathrm{e}}\end{array}\right.$

where $Y_{i}$ is the mass fraction, $\dot{w}_{i}=M_{i} P_{i j} \dot{\mathrm{W}}_{j}$ the reaction rate and $\mathrm{M}_{\mathrm{i}}$ the molar mass of specie $\mathrm{i}$, and $\dot{\mathrm{w}}_{\mathrm{j}}$ is the rate of reaction of reaction $\mathrm{j}, \sigma$ the thermal radiation term, $\tau^{\mathrm{k}}$ the truncation error associated with the numerical algorithm, with $\mathrm{k}=\left\{\rho, \mathrm{Y}_{\mathrm{i}}, \mathrm{v}, \mathrm{e}\right\}$, and,

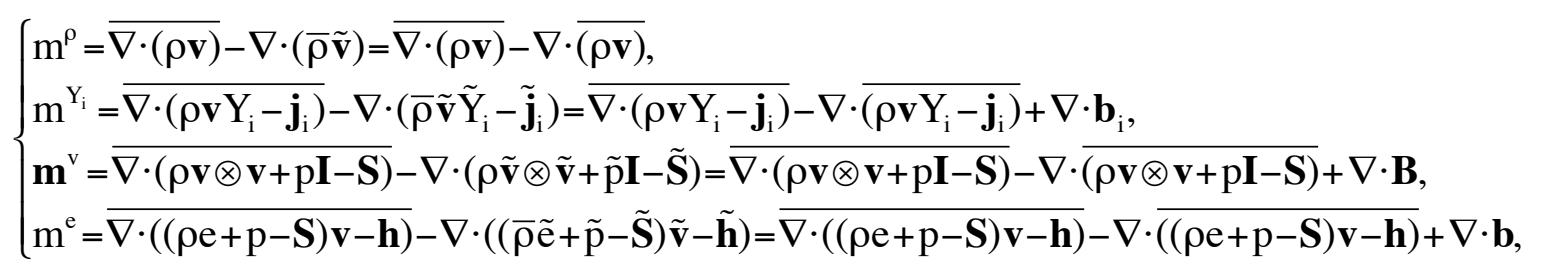

where $\mathbf{B}=\bar{\rho}(\overline{\mathbf{v} \otimes \mathbf{v}}-\tilde{\mathbf{v}} \otimes \tilde{\mathbf{v}})+\tilde{\mathbf{S}}-\overline{\mathbf{S}}+(\overline{\mathrm{p}}-\tilde{\mathrm{p}}) \mathbf{I}, \quad \mathbf{b}_{\mathrm{i}}=\overline{\boldsymbol{\rho}}\left(\overline{\mathbf{v} Y_{i}}-\tilde{\mathbf{v}} \tilde{Y}_{i}\right)+\tilde{\mathbf{j}}_{i}-\overline{\mathbf{j}}_{\mathrm{i}}$ and $\mathbf{b}=\bar{\rho}(\overline{\mathbf{v e}}-\tilde{\mathbf{v}} \tilde{\mathbf{e}})+\tilde{\mathbf{S}} \tilde{\mathbf{v}}-\overline{\mathbf{S v}}+\overline{\mathrm{p} \mathbf{v}}-\overline{\mathrm{p}} \tilde{\mathbf{v}}+\tilde{\mathbf{h}}-\overline{\mathbf{h}}$. In addition, the reaction rates $\dot{\mathrm{w}}_{\mathrm{i}}$ and the constitutive equations $\left(\mathrm{p}, \mathbf{S}, \mathbf{h}\right.$ and $\mathbf{j}_{\mathrm{i}}$ ) are usually highly non-linear functions of the dependent variables. Of particular interest is the reaction rate that can be decomposed as $\overline{\dot{\mathrm{w}}}_{\mathrm{i}}=\overline{\dot{\mathrm{w}}\left(\rho, \mathrm{Y}_{\mathrm{i}}, \mathrm{T}\right)_{\mathrm{i}}}=\tilde{\dot{\mathrm{w}}}_{\mathrm{i}}+\left[\overline{\dot{\mathrm{w}}}_{\mathrm{i}}-\tilde{\dot{\mathrm{w}}}_{\mathrm{i}}\right]=\tilde{\dot{\mathrm{w}}}_{\mathrm{i}}+\dot{\mathrm{W}}_{\mathrm{i}, \mathrm{ggg}}$, where $\tilde{\dot{\mathrm{w}}}_{\mathrm{i}}=\dot{\mathrm{w}}_{\mathrm{i}}(\bar{\rho}, \tilde{\mathrm{Y}} \mathrm{i}, \tilde{\mathrm{T}})$. For the fluid dynamic part, closure models are typically borrowed from the incompressible case, and corrected for the variable density, whereas for the chemistry part, and the reaction rates in particular, other approaches are necessary. First, the number of chemical reaction has to be limited, and reduced or global mechanisms will be used instead of detailed mechanisms, which diminish the ability to predict minor species, which in turn affects the accuracy of the reaction rates. Second, the nonlinearity of the reaction rates (typically in Arrhenius form) makes the closure modeling of $\dot{\mathrm{w}}_{\mathrm{i}, \mathrm{ggs}}$ very difficult, [48]. Instead of following this route other models, such as flamelet models, lineareddy models, probability density function models, [49], are commonly used. Here we compare predictions from a flamelet model, [50], and an ILES model, [51], in order to emphasize differences, similarities and the robustness of the LES concept. 
In the ILES model the thermodynamic model consists of the equation-of-state for an ideal gas mixture and the equation-of-state for the enthalpy of the mixture. Diffusive transport properties i.e. species diffusion (described by a Fickian scheme), mixture viscosity (modeled by Sutherland's law), and thermal conduction (modeled similarly) are included, whilst thermal diffusion is neglected. Finite-rate chemistry is included through the two-step mechanism, [52]. The time integration is performed by a predictor-corrector method, viscous and diffusive terms are handled using central differencing whereas convective terms are represented by a Flux Corrected Transport (FCT) scheme, [15]. The implicit or built-in subgrid models therefore becomes $\mathbf{b}_{\mathrm{i}} \approx \rho \mathbf{C} \nabla \tilde{\mathrm{Y}}_{\mathrm{i}}$, $\mathbf{B} \approx \rho\left(\mathbf{C}(\nabla \tilde{\mathbf{v}})^{\mathrm{T}}+(\nabla \tilde{\mathbf{v}}) \mathbf{C}^{\mathrm{T}}\right.$ and $\mathbf{b} \approx \rho \mathbf{C} \nabla \tilde{\mathrm{e}}$, where $\mathbf{C}=\chi(\tilde{\mathbf{v}} \otimes \mathbf{d}), \chi=\frac{1}{2}(1-\Psi)\left(\beta^{-}-\beta^{+}\right)$and $\beta^{ \pm}=\frac{1}{2}(\operatorname{sign}(\tilde{\mathbf{v}}) \pm 1)$. For this model the reaction rate is given by $\overline{\dot{\mathrm{w}}}_{\mathrm{i}} \approx \tilde{\dot{\mathrm{W}}}_{\mathrm{i}}$ such that $\dot{\mathrm{W}}_{\mathrm{i}, \mathrm{sg}}=0$.

In the flamelet LES model, the flamelet assumption allows decoupling the chemistry from the fluid mechanics which enables pre-computing the chemistry as parameterized by the passive and reactive scalars $\mathrm{z}$ and $\zeta$ using the flamelet library $\mathrm{Y}_{\mathrm{i}}=\hat{\mathrm{Y}}_{\mathrm{i}}(\mathrm{z}, \zeta)$. The thermodynamic and gas mixture models are the same as for the ILES model but with equal diffusivities, D. The modified equations of the flamelet LES model are generally consistent with (5) with the exception of the species equations being replaced by the flamelet equations,

$\left\{\begin{array}{l}\partial_{\mathrm{t}}(\bar{\rho} \tilde{z})+\nabla \cdot(\bar{\rho} \tilde{\mathbf{v}} \tilde{z})=\nabla \cdot(D \nabla \tilde{z})+\tau^{\mathrm{z}}-\mathrm{m}^{\mathrm{z}}, \\ \partial_{\mathrm{t}}(\bar{\rho} \tilde{\zeta})+\nabla \cdot(\bar{\rho} \tilde{\mathbf{v}})=\nabla \cdot(\mathrm{D} \nabla \tilde{\zeta})-\overline{\dot{\mathrm{w}}_{\zeta}}+\tau^{\zeta}-\mathrm{m}^{\zeta}\end{array}\right.$

where,

$$
\left\{\begin{array}{l}
\mathrm{m}^{\mathrm{z}}=\overline{\nabla \cdot(\rho \mathbf{v z}-\mathrm{D} \nabla \mathrm{z})}-\nabla \cdot(\bar{\rho} \tilde{\mathbf{v}} \tilde{z}-\mathrm{D} \nabla \tilde{z})=\overline{\nabla \cdot(\rho \mathbf{v z}-\mathrm{D} \nabla \mathrm{z})}-\nabla \cdot \overline{(\rho \mathbf{v z}-\mathrm{D} \nabla \mathrm{z})}+\nabla \cdot \mathbf{b}_{\mathrm{z}} \\
\mathrm{m}^{\zeta}=\overline{\nabla \cdot(\rho \mathbf{v} \zeta-\mathrm{D} \nabla \xi)}-\nabla \cdot(\bar{\rho} \tilde{\mathbf{v}} \zeta-\mathrm{D} \nabla \tilde{\xi})=\overline{\nabla \cdot(\rho \mathbf{v} \zeta-\mathrm{D} \nabla \zeta)}-\nabla \cdot \overline{(\rho \mathbf{v} \zeta-\mathrm{D} \nabla \xi)}+\nabla \cdot \mathbf{b}_{\xi}
\end{array}\right.
$$

and $\dot{\mathrm{w}}_{\mathcal{\zeta}}$ is the reaction-rate. To close the subgrid terms we employ the Mixed Model (MM), [42],

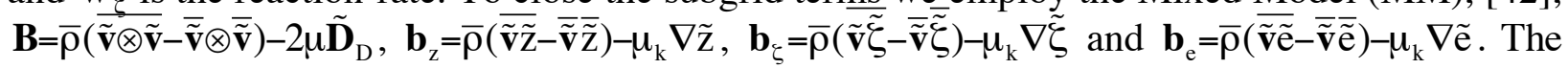
flamelet model uses an explicit filtering approach, $[50,53]$, in which $\dot{\mathrm{w}}_{\zeta}$ can be represented by a Dirac function scaled to preserve the correct reaction rate, $\dot{\mathrm{w}}_{\zeta}=\rho_{\mathrm{u}} S_{\mathrm{u}} \delta(\mathbf{x}-\hat{\mathbf{x}})$, where $\hat{\mathbf{x}}$ denotes the location of the flame. Normal to the flame, in a coordinate system attached to, and moving with, the flame front this implies that $\overline{\dot{\mathrm{w}}}_{\zeta}$ can be expressed in terms of $\dot{\mathrm{w}}_{\zeta}=\rho_{\mathrm{u}} \mathrm{S}_{\mathrm{u}} \delta(\mathrm{s})$ and the Gaussian filter kernel, having the width $\Delta$, which implies that $\overline{\dot{\mathrm{w}}_{\zeta}}=\rho_{\mathrm{u}} S_{\mathrm{u}} \sqrt{\frac{6}{\pi}} \Delta^{-1} \exp \left(-6 \mathrm{~s}^{2} / \Delta^{2}\right)$. Solving a 1D version of $\left(7_{2}\right)$, i.e. $\partial_{\mathrm{s}}(\tilde{\zeta})-\partial_{\mathrm{s}}\left(\partial_{\mathrm{s}}(\tilde{\zeta}) / \mathrm{a}\right)=\sqrt{\frac{6}{\pi}} \exp \left(-6 \mathrm{~s}^{2}\right)$, where $\mathrm{a}=\rho_{\mathrm{u}} \mathrm{S}_{\mathrm{u}} \Delta / \mathrm{D}=\Delta / \delta_{\mathrm{L}}^{0}$, we obtain the intrinsic low-pass filtered flame structure that in turn can be used to construct a flamelet library, $\Pi$, for the reaction rate in terms of $\tilde{\zeta}$ and the parameter a, such that $\Pi=\Pi(\tilde{\zeta}, a),[53]$. In order to ensure the correct flame propagation speed, and for consistency, we must also require that $D=\rho_{u} S_{u} \Delta / a$, such that the following modeled $\tilde{\zeta}$-equation results,

$$
\partial_{\mathrm{t}}(\bar{\rho} \tilde{\zeta})+\nabla \cdot(\bar{\rho} \tilde{\mathbf{v}} \tilde{\zeta})=\nabla \cdot\left[\left(\rho_{\mathrm{u}} \mathrm{S}_{\mathrm{u}} \Xi \Delta / \mathrm{a}\right) \nabla \tilde{\xi}\right]-\left(\rho_{\mathrm{u}} \mathrm{S}_{\mathrm{u}} \Xi / \Delta\right) \Pi(\tilde{\zeta}, \mathrm{a})
$$

A fractal flame wrinkling model, [54], is used to model $\Xi$, which is interpreted as the ratio 
between the wrinkled and laminar flame surface, $\Xi=\left(\Delta / \varepsilon_{\mathrm{i}}\right)^{\mathrm{D}-2}$, where $\varepsilon_{\mathrm{i}}$ is the inner cut-off and D the fractal dimension. $\varepsilon_{\mathrm{i}}$ is defined as the subgrid wrinkling length scale, evaluated from the curvature tensor, so that $\varepsilon_{\mathrm{i}}=\mathrm{S}_{\mathrm{u}}^{0} \Delta / \mathrm{v}^{\prime} \Gamma$, where $\Gamma$ is the efficiency function, [55], taking the straining of all scales smaller than $\Delta$ so that $\Xi=\left(\Gamma v^{\prime} / S_{u}^{0}\right)^{\mathrm{D}-2}$ into account, where $\mathrm{v}^{\prime}=\sqrt{2 \mathrm{k} / 3}$ is the subgrid velocity and $S_{\mathrm{u}}^{0}$ is the unstretched laminar flame speed. Following [56], the fractal dimension is parameterized as $\mathrm{D}=2.00 /\left(\mathrm{v}^{\prime} / \mathrm{S}_{\mathrm{u}}^{0}+1\right)+2.35 /\left(\mathrm{S}_{\mathrm{u}}^{0} / \mathrm{v}^{\prime}+1\right)$. We also assume that the inner structure of the reaction zone is virtually unaffected by the turbulence such that $\left\langle\rho S_{u}\right\rangle \approx \rho_{u} S_{u}(\tilde{z})$.

The time integration is performed by the same predictor-corrector method as used for ILES whereas convective, viscous and diffusive terms are treated using central differencing.

The ILES finite rate chemistry model and the LES flamelet models are evaluated using the VOLVO Validation Rig, [57], which involves a rectilinear combustor of length L $=25 \mathrm{~h}$, height $3 \mathrm{~h}$ and width $6 \mathrm{~h}$ in which a triangular flameholder, with height $\mathrm{h}=0.04 \mathrm{~m}$ is located at $\mathrm{x} / \mathrm{h}=7.95$. Optical access is provided by quartz windows at the sides of the combustor whereas the upper and lower walls are water cooled. The computational domain employs $\sim 2$ million grid points being periodic in the spanwise direction, with a width of 3h. At the inlet, Dirichlet conditions are used for all variables besides $p$ for which zero Neumann conditions are used, and at the outlet, wavetransmissive conditions are used. For all walls no-slip conditions are used. The Validation Rig was operated under a variety of conditions, and here we focus on a premixed case, using $\mathrm{C}_{3} \mathrm{H}_{8}$ as fuel, with $\mathrm{v}_{\text {in }}=17 \mathrm{~m} / \mathrm{s}, \mathrm{T}_{\text {in }}=298 \mathrm{~K}$ and an equivalence ratio of $\phi=0.62$.

The flame anchors behind the flameholder due to the recirculation of hot combustion products in the wake behind the bluff-body, and spanwise vortices are shed off the upper and lower edges of the prism, figure 7 . The symmetric shedding and roll-up results in longitudinal vortices stretched between succeeding spanwise vortices on either side of the centerline. As the flame is convected downstream it propagates normal to itself, causing negatively curved wrinkles to contract and positively curved wrinkles to expand. The high-temperature region is associated with exothermicity, and burning occurs at the fuel-rich side of the shear layers, where also most of the mixing between cold reactants and hot products takes place.
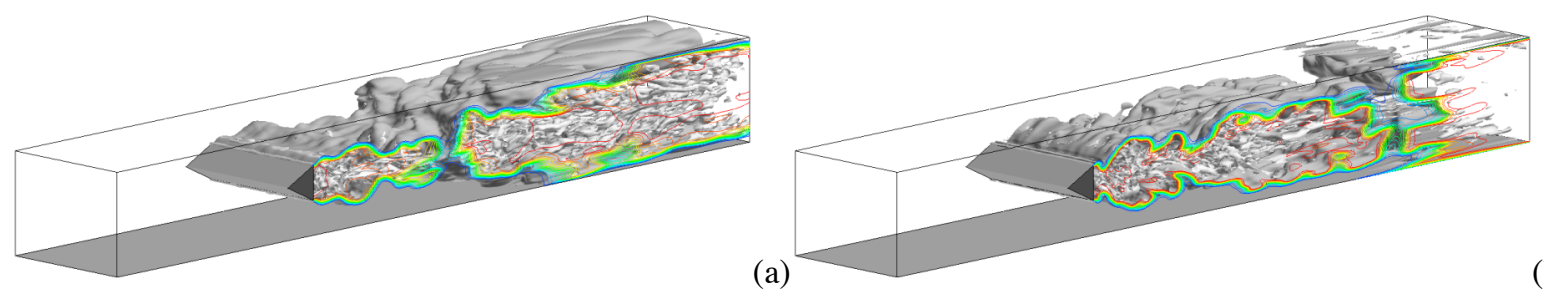

(b)

Figure 7. Combustion. Instantaneous perspective views of the flame and the vorticity in terms of an iso-surface of the reaction coordinate, contours of the temperature and an iso-surface of $\lambda_{2}$, being a good quantifier of the vorticity from (a) the finite rate chemistry ILES and (b) the flamelet LES.

Figure 8 shows a comparison of the time-averaged axial velocity $\left\langle\tilde{\mathrm{v}}_{\mathrm{x}}\right\rangle$ and the temperature $\langle\tilde{T}\rangle$ at three axial locations between the finite rate chemistry ILES and the flamelet LES. For $\left\langle\tilde{v}_{x}\right\rangle$ we find that both LES models generally result in adequate agreement with the measurement data. At $\mathrm{x} / \mathrm{h}=8.7$ the measurement data show a flattened velocity profile that is not well captured 
by any of the models. In the recovery region and further downstream (at $\mathrm{x} / \mathrm{h}=11.5$ and 16.5$)$ the agreement is satisfactory, but with a tendency for the ILES finite-rate chemistry model to predict a somewhat too long recirculation region, and a tendency for the LES flamelet model to overpre$\operatorname{dict}\left\langle\tilde{\mathrm{v}}_{\mathrm{x}}\right\rangle$ in the core of the combustion duct at $\mathrm{x} / \mathrm{h}=16.5$. For $\langle\tilde{\mathrm{T}}\rangle$ there are two sets of measurements: CARS and gas analysis, [58], which give slightly different profiles at $\mathrm{x} / \mathrm{h}=11.5$ and 16.5. It is generally believed that the CARS data is more accurate than the gas analysis data since the samples continue to react after being extracted from the combustor, and therefore we primarily compare with the CARS data. It seems that the LES flamelet model results in a somewhat more narrow temperature distribution than the ILES finite rate chemistry model. The peak temperature is well captured by all models, indicating that also $\mathrm{NO}_{\mathrm{x}}$ emissions would be reasonably well predicted. Considering however, the complexity of the laboratory rig and the sudden expansion-type outlet with water-mist cooling, not incorporated in the computational model, the agreement between LES and ILES, and experimental data is satisfactory.
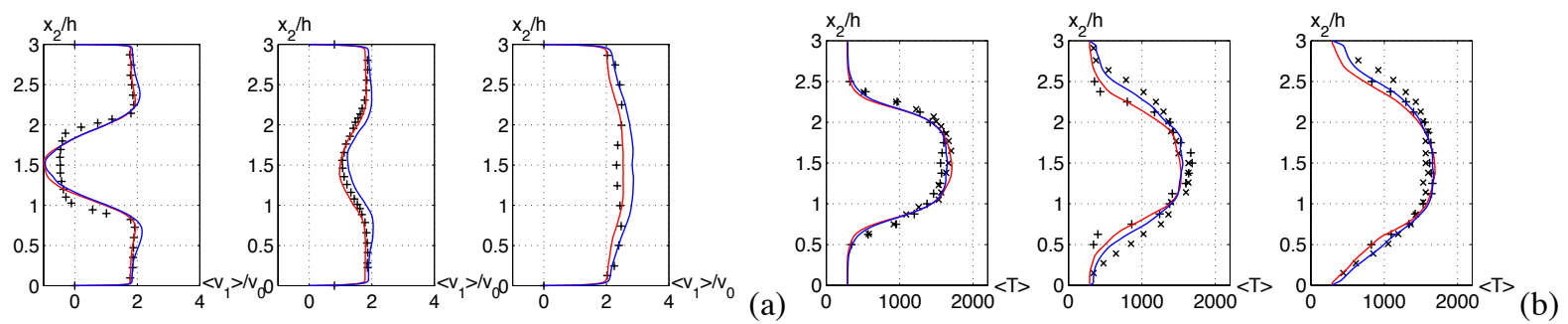

Figure 8. Combustion. Time-averaged (a) axial velocity and (b) temperature for the VOLVO Validation Rig case using the finite rate chemistry ILES (blue) and the flamelet LES models (red). Experimental data is denoted by symbols $(+)$ for LDV and CARS and $(x)$ for gas analysis.

\section{CAVITATION}

Cavitation causes detrimental effects like loss of thrust, noise and vibration and erosion on fluid machineries such as pumps, water turbines and marine propellers. Cavitation is a two-phase flow phenomenon associated with vaporization and condensation. Cavitation is similar to boiling, the difference being that boiling is due to heating, whereas cavitation is caused by the increase in the velocity resulting in pressures lower than the vapor pressure. The basic fluid machinery component experiencing cavitation is the hydrofoil - the design of which is a trade-off between highperformance and cavitation generation. Appearances of cavitation differ substantially according to flow and foil conditions even if the foil is the same, figure 9. When the angle of attack is small and the boundary layer along the foil does not separate, bubble cavitation generates on the suction side of the foil. The bubble cavitation generates from small air-bubbles in the flow, which are called nuclei, and if removed bubble cavitation will not occur. Increase of the angle of attack causes separation of the boundary layer, and other type of cavitation occurs on the foil. This type of cavitation is called sheet cavitation in which the cavity appears fixed on the foil. Lowering the static pressure at the same angle of attack, the sheet becomes longer and unstable. Cloud cavitation is generated periodically from the rear part of a sheet cavity, and often causes severe erosion and noise. Finite span foils generate also another type of cavitation, tip-cavitation, which stems 
from the low-pressure region of the vortex core emanating from the tip of the foil. Supercavitation is when the entire body becomes engulfed by the cavity.
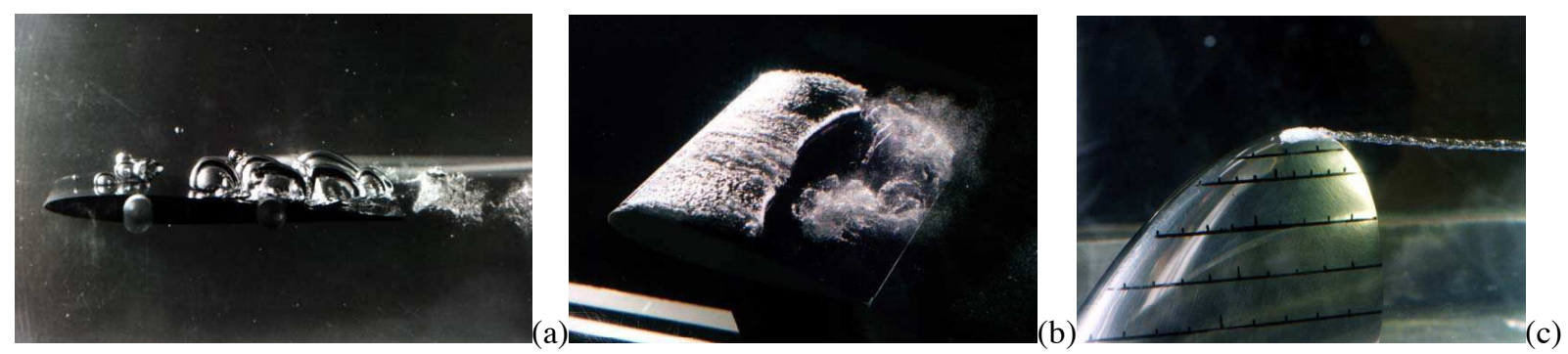

Figure 9. Cavitation. (a) Bubble cavitation, (b) sheet cavitation with emerging cloud cavitation and (c) tip-vortex cavitation. Images from http://www.fluidlab.naoe.t.u-tokyo.ac.jp.

Classical modeling of cavitation uses linear or non-linear models using boundary-element methods, [59]. If a more accurate model is required that contains more physics and that can be applied to a wider class of problems than foils, the model has to be based on the NSE. Studying cavitation by means of the NSE includes the turbulent flow, the sharp and complex interface between the gaseous and liquid phases and the modeling of vaporization and condensation. The flow is best handled with unsteady models, such as DES or LES, although RANS have been used for cavitation predictions in the past, e.g. [60]. DES has to the authors knowledge not been used for cavitation predictions as of today, whereas LES have been used by some investigators, [6162]. LES is by far the most versatile, but its use in cavitation is not strightforward. The interface can be handled in many ways: Marker methods depend on Lagrangian tracking of virtual particles, [63]. Moving mesh methods track the interface by adapting the grid to the location of the interface as a given grid-line, [64]. Volume of fluid techniques is an Eulerian interface capturing method, [65], in which a scalar field, advected by the flow, describes the ratio of vapor to liquid at every point, so that liquid is defined by $\gamma=1$ whereas pure is defined by $\gamma=0$. The phase change is even more challenging and subject to empiricism. Spherical bubble dynamics, as described by the Rayleigh-Plesset equation, [60], is a simple but still attractive way of determining the rate of phase change. With this method the Rayleigh-Plesset equation has to be solved at each time step for a given number of distributed nuclei. The total volume change of nuclei in a control volume defines the mass transfer between vapor and liquid. As the liquid phase is virtually incompressible and the compressible effects are limited to inside the cavity, it is reasonable to use a variable density formulation of the NSE. Following [23] the finite volume formulation of the low-pass filtered equations or the raw equations results in the modified equations,

$\left\{\begin{array}{l}\nabla \cdot \bar{v}=\dot{m}\left(\rho_{1}^{-1}-\rho_{g}^{-1}\right)+\tau^{\rho}, \\ \partial_{t}(\rho \overline{\mathbf{v}})+\nabla \cdot(\rho \overline{\mathbf{v}} \otimes \overline{\mathbf{v}})=-\nabla \bar{p}+\nabla \cdot \overline{\mathbf{S}}+\overline{\mathbf{f}}_{\sigma}+\tau^{\mathrm{v}}+\mathbf{m}^{\mathrm{v}}, \\ \partial_{\mathrm{t}}(\gamma)+\nabla \cdot(\overline{\mathbf{v}} \gamma)=-\left(\rho_{\mathrm{g}} /\left(\rho_{1}-\rho_{\mathrm{g}}\right)\right) \nabla \cdot \overline{\mathrm{v}}+\tau^{\gamma}+\mathrm{m}^{\gamma},\end{array}\right.$

where $\dot{m}=\dot{m}^{+}+\dot{m}^{-}$is the interphase mass transfer rate, [62], $\rho=\gamma \rho_{1}+(1-\gamma) \rho_{\mathrm{g}}$ the density, where sub- 
scripts 1 and $g$ denote the liquid and gas phases, respectively, $\mathbf{S}=2 \mu \mathbf{D}$ the viscous stress tensor, $\mu=\gamma \mu_{1}+(1-\gamma) \mu_{\mathrm{g}}$ the viscosity, $\mathbf{f}_{\sigma}$ the surface tension force, $\tau^{\mathrm{k}}$ the truncation error, and $\mathrm{m}^{\mathrm{i}}$ the unresolved transport terms. The unresolved transport terms that occur in LES include terms that are very difficult to model, and for which no indications are available of how to close these terms and in the light of this we only use ILES for the cavitation studies.

To examine the possibilities of simulating cavitation we study the flow and cavitation over a NACA0012 wing profile with chord length $\mathrm{C}=0.14 \mathrm{~m}$. The size of the computational domain is $15 \mathrm{C} \times 10 \mathrm{C} \times 5 \mathrm{C}$ with the following boundary conditions: At the inlet, velocity and volume fraction is fixed whereas the pressure satisfies a Neumann condition. The reverse is valid for the outlet, at which the pressure is fixed. The wing surface consists of a no-slip Dirichlet and Neumann conditions for pressure and volume fraction, respectively, whereas periodic conditions are imposed in the spanwise direction. An inlet velocity of $\mathrm{v}_{0}=10 \mathrm{~m} / \mathrm{s}$ is chosen to match the experiments, [66], whereupon ambient pressure $\mathrm{p}_{0}$ and vapor pressure $\mathrm{p}_{\mathrm{v}}$, are chosen to give a cavitation number of $\sigma=1.0$, resulting in that $\mathrm{p}_{\mathrm{v}}=2.3 \mathrm{kPa}$ and $\mathrm{p}_{0}=52.3 \mathrm{kPa}$. During the simulation the wing boundary rotates inside the domain, and the mesh is correspondingly deformed.
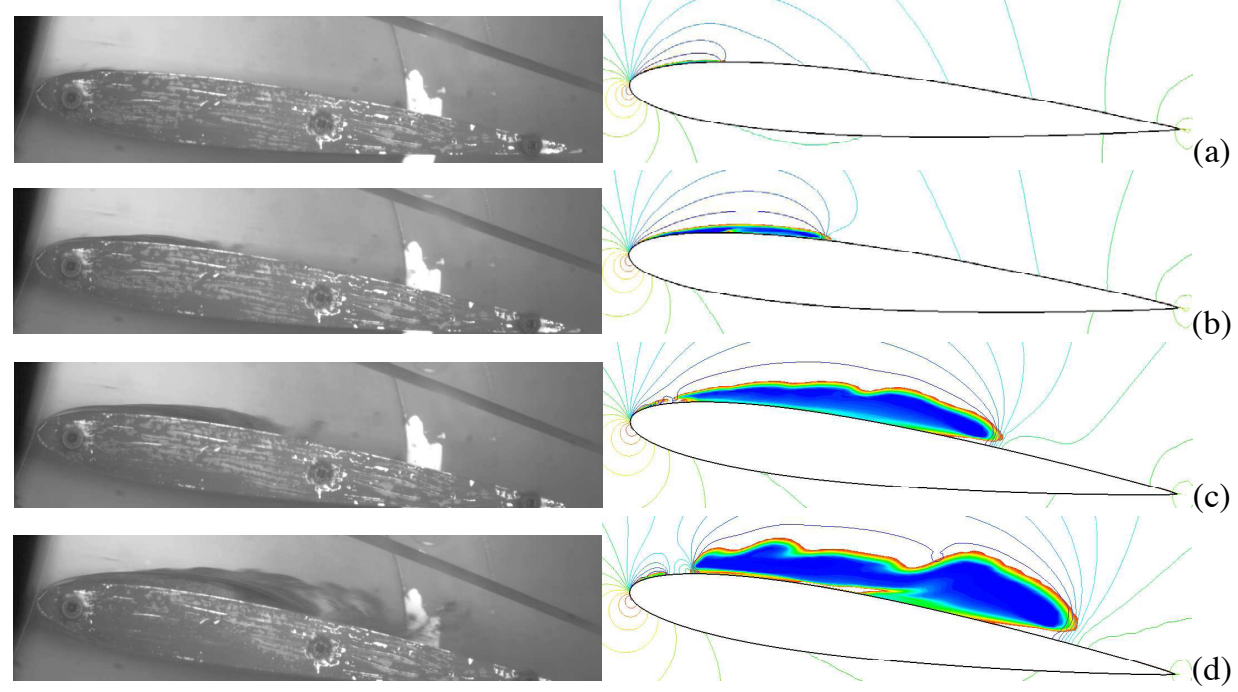

Figure 10. Cavitation. Sheet cavity growth as the angle of attack of the wing, $\alpha$, increase. (a) $\alpha=4.7^{\circ}$, (b) $\alpha=5.7^{\circ}$, (c) $\alpha=7.7^{\circ}$ and (d) $\alpha=8.7^{\circ}$.

Ocular analysis of the side view movie from the experiments shows that the first occurrence of cavitation takes place at $\alpha \approx 5.0^{\circ}$, whereas this occurs at $\alpha \approx 4.3^{\circ}$ in the simulation model, that is considered reasonable considering the complexity of the problem. Would for example the cavitation tunnel water contain a large amount of nuclei so that cavitation occurs exactly at the vapor pressure, one may come close but this is seldom the case. A rapid growth of a thin sheet cavity follows the inception as seen in figure 10. From these images it is clear that the simulated cavity growth is somewhat too rapid, and thus starts shedding from the leading edge earlier than in the experiments. Comparing the sequences in figure 10 shows that whereas the model predicts a thin interface between the vapor and liquid the experiments show a cloudy cavity with a diffuse 
boundary. Using the present model, cloud cavities cannot be properly represented due to intrinsic properties of the cavitation model and its inherent links to the numerical schemes that automatically preserves a thin as possible interface.

\section{CONCLUDING REMARKS}

In the absence of an accepted universal theory of turbulence, the development of improved CFD models for engineering applications are unavoidably pragmatic and based on the use of empirical information. When complex physics is involved, as often is the case for engineering applications, this issues becomes increasingly important since often the turbulence affects the rates of production of noise, chemical species etc. The alternatives to LES or ILES are DES or RANS, of which the latter has many well-known deficiencies and limitations. Regarding DES sufficient information is not yet available for making proper decisions. In this study we have compared conventional LES and ILES for incompressible turbulent flows, compressible flows, aerodynamic noise, combustion and cavitation. Typically, this involves modeling also other quantities than the conventional subgrid stress tensor or flux vectors, such as reaction rates or noise sources. The modeling of these quantities has here been performed in a manner consistent with the LES or ILES formalism. For combustion we for example employ a flamelet LES model. The models are formulated in a finite volume setting and are presented in terms of the modified equations, involving not only the unresolved transport terms (for LES) but also the commutation error and the leading order truncation error terms. For the subgrid model, it is familiar that the implicit subgrid model is of a generalized eddy viscosity type, the intrinsic details of which are dependent on the flux limiter that is employed in the flux reconstruction step of the high-resolution non-oscillatory schemes typically used in ILES. Both ILES and LES typically give good results when compared with experimental data, underlining the robustness of the LES approach. Further challenges for LES and ILES include constructing a common appropriate mathematical and physical framework for its analysis and development and concepts for incorporating more physics into the overall scheme to improve the global performance of the model.

\section{ACKNOWLEDGEMENT}

The author whishes to express his appreciation to N. Alin,, M. R. Bensow, M. Berglund, C. Dutton, C. Duwig, F. Grinstein, M. Liefendahl, O. Parmhed, T. Persson, U. Svennberg and N. Wikström for providing experimental and computational data and for many good discussions. The author also which to acknowledge the financial support from the Swedish Armed Forces.

\section{REFERENCES}

[1] Pope, S.B.; 2000, “Turbulent Flows”, Cambridge University Press.

[2] Launder, B.E. \& Spalding, D.B.; 1972, Mathematical Models of Turbulence, Academic Press.

[3] Wilcox, D.C.; 1993, “Turbulence Modeling for CFD”, DCW Industries

[4] Ferziger J.H. \& Leslie D.C.; 1979, "Large Eddy Simulation - A Predictive Approach to Turbulent Flow Computation", AIAA 79-1441.

[5] Boris, J.P., Grinstein, F.F., Oran, E.S. \& Kolbe, R.L.; 1992, "New Insights into Large Eddy Simulation”, Fluid Dyn. Res., 10, p 199. 
[6] Sagaut P.; 2001, "Large Eddy Simulation for Incompressible Flows”, Springer Verlag.

[7] Boris, J.P.; 1992, “On Large Eddy Simulations Using Subgrid Turbulence Models”, in Wither Turbulence? Turbulence at the Crossroads, Ed. Lumly J.L., Lecture Notes in Physics, 357, Springer Verlag, Berlin, p 344.

[8] Nikitin N.V., Nicoud F. Wasistho B. Squires K.D. \& Spalart P.R.; 2000, “An Approach to Wall Modelling in Large Eddy Simulation", Phys. Fluids, 12, p 1629.

[9] Lourenco L.M. \& Shih C.; 1993, "Characteristics of the Plane Turbulent Near Wake of a Circular Cylinder, A Particle Image Velocimetry Study”. Data taken from Ma X., Karamanos G.S. \& Karniadakis G.E., 2000, "Dynamics and Low-Dimensionality of a Turbulent Near Wake”, J. Fluid Mech., 410, p 29.

[10] Ong J. \& Wallace L.; 1996, "The Velocity Field of the Turbulent Very Near Wake of a Circular Cylinder", Exp. in Fluids, 20, p 441.

[11] Cantwell B. \& Coles D.; 1983, “An Experimental Study of Entrainment and Transport in the Turbulent Near Wake of a Circular Cylinder”, J. Fluid Mech., 136, p 321.

[12] Persson T., Fureby C. \& Bensow R.; 2005, "Large Eddy Sim ulation and Deatched Eddy Simulation of Turbulent Flow around a Circular Cylinder", Report No 05:98.

[13] Byun G. \& Simpson R.L.; 2005, "Structure of Three-dimensional Separated Flow on a Axisymmetric Bump", AIAA 05-0113.

[14] Persson T. M. Liefevendahl, Bensow R. \& Fureby C., 2005, "Numerical Investigation of the Flow over an Axisymmetric Hill using LES, DES and RANS", J. Turbulence, 7, 4.

[15] Oran S.S. \& Boris J.P.; 2000, “Numerical Simulation of Reactive Flow”, Cambridge University Press.

[16] Chomiak J. \& Nisbeth J.; 1995, "Modeling Variable Density effects in Turbulent Flames - Some basic Considerations", Comb. \& Flame, 102, p 371.

[17] Grinstein F.F. \& Fureby C.; 2004, "From Homogeneous Turbulent Flows to Pollutant Dispersion in Urban Areas: Recent Progress in Monotonically Integrated LES”, Computing in Science and Engineering, March/April issue, p 36.

[18] Grinstein F.F., Margolin L. \& Rider B.; 2006, "Implicit Large Eddy Simulation: Computing Turbulent Fluid Dynamics", Cambridge University Press. In Press

[19] Groves, N.C., Huang, T.T. \& Chang, M.S.; 1989, "Geometric Characteristics of DARPA SUBOFF Models", Report DTRC/SHD-1298-01.

[20] Huang T.T., Liu H-L., Groves N.C., Forlini T.J., Blanton J. \& Gowing S.; 1992, "Measurements of Flows over an Axisymmetric Body with Various Appendages (DARPA SUBOFF Experiments)”, Proc. 19th Symp. on Naval Hydrodynamics, Seoul, Korea.

[21] Persson T., Bensow R., Fureby C., Alin N., Svennberg U.; 2004, "Large Eddy Simulation of the Viscous Flow around Submarine Hulls”, Proc. $25^{\text {th }}$ Symp. on Naval Hydrodynamics, p 261, St. John's, Canada.

[22] Fureby C., Alin N., Wikström N., Menon S., Persson L. \& Svanstedt N.; 2004, "On Large Eddy Simulations of High Re-number Wall Bounded Flows", AIAA.J. 42, p 457

[23] Grinstein F.F. \& Fureby C.; 2002, “Recent Progress on MILES for High Re Flows”, J. Fluids Engng., 124, p 848.

[24] Schumann U.; 1975, "Subgrid Scale Model for Finite Difference Simulation of Turbulent Flows in Plane Channels and Annuli, J. Comp. Phys., 18, p 376.

[25] Goldstein R. J.; 1996, "Fluid Mechanics Measurements", Taylor \& Francis, Washington, D.C.

[26] Li D.Q.; 1994, "Investigation of Propeller Rudder Interaction b y Numerical Methods", PhD Thesis, Chalmers University of Technology, Sweden.

[27] Hardin J.C. \& Pope D.S.; 1994, “An Acoustic/Viscous Splitting Technique for Computational Aeroacoustics”, J. Theory Comp Fluid Dyn, 6, p 323.

[28] Lighthill M.J.; 1952, “On Sound Generated Aerodynamically: I General Theory”, Proc. Royal Soc., 211.

[29] Ffowkcs-Williams J.E. \& Hawkings D.L.; 1969, "Sound Generation by Turbulence and Surfaces in Arbitrary Motion”, Phil. Trans. Royal Society, 264, No 1151.

[30] Lyrintzis A.S.; 1994, "Review: The use of Kirchhoff's Method in Computational Aeroacoustics", J. Fluids Engng, 116, p 665. 
[31] Höld R., Brenneis A., Eberle A., Schwarz V. \& Siegert R.; 1999, "Numerical Simulation of Aeroacoustic Sound Generated by Generic Bodies Placed on a Plate: Part 1 - Prediction of Aerodynamic Sources", AIAA 991896.

[32] Siegert R., Schwarz V. \& Reichenberger J.; 1999, "Numerical Simulation of Aeroacoustic Sound Generated by Generic Bodies Placed on a Plate: Part 1 - Prediction of Radiated Sound Pressure”, AIAA 99-1895.

[34] Delery J. \& Lacau R.G.; 1987, "Prediction of Base Flows", AGARD Report 654.

[35] Putnam L.E. \& Bissinger N.C.; 1985, "Results of AGARD Assessment of Prediction Capabilities for Nozzle Afterbody Flows", AIAA 85-1464.

[36] Sahu J.; 1994, "Numerical Computations of Supersonic Base Flow with Special Emphasis on Turbulence Modelling”, AIAA J., 32, p 1547.

[37] Fureby C., Nilsson Y. \& Andersson K.; 1999, "Large Eddy Simulation of Supersonic Base Flow“, AIAA 990537.

[38] Forsythe J.R., Hoffmann K.A \& Squires K.D.; 2002, "Detatched-Eddy Simulation with Compressibility Corrections Applied to a Supersonic Axisymmetric Base Flow", AIAA 02-0586.

[39] Simon F., Deck P., Guillen P. \& Cayzac R.; 2006, "Numerical Simulations of Projectile Baseflow", AIAA 06-1116.

[40] Herrin J.L. \& Dutton C.J.; 1994, "Supersonic Base Flow Experiments in the Near Wake of A Cylindrical Afterbody", AIAA.J., 32, p 77.

[41] Mathur T. \& Dutton J.C.; 1996, "Base-Bleed Experiments with a Cylindrical Afterbody in Supersonic Flow", J. Spacecrafts \& Rockets, 33, p 30.

[42] Bardina J., Ferziger J.H, \& Reynolds W.C.; 1980, "Improved Subgrid Scale Models for Large Eddy Simulations", AIAA 80-1357.

[43] van-Leer B.; 1974, "Towards the Ultimate Conservative Differencing Scheme V. A second order Sequel to Gudonovs Method”, J. Comp. Phys., 32, p 101.

[44] Boris J.P. \& Book D.L.; 1973, "Flux-corrected Transport I. SHASTA, a Fluid Transport Algorithm that Works", J. Comp. Phys, 11, p 38.

145] Gottlieb S. \& Shu C.-W.; 1998, "Total Variational Diminishing Runge-Kutta Schemes”, Mathematics of Computation, 67, p 73.

[46] Kohse-Höinghaus K, Barlow R.S., Aldén M. \& Wolfrum J.; 2005, "Combustion at the Focus: laser Diagnostics and Control”, Proc. Comb, Inst, 30, p 89.

[47] Vervish L., Domingo P. \& Hauguel R.; 2003, "Turbulent Combustion in the Light of Direct and Large Eddy Simulation", Turbulent Shear Flow Phenomena 3, Sendai, Japan.

[48] Libby P.A. \& Williams F.A.; 1980, "Fundamental Aspects", In Turbulent Reactive Flows, Eds. Libby P.A. \& Williams F.A., Springer Verlag, Berlin, p 1.

[49] Poinsot T. \& Veynante D.; 2001, “Theoretical and Numerical Combustion”, Edwards.

[50] Fureby C.; 2006, "A Comparison of Flamelet Models for Premixed Turbulent Combustion”, AIAA 06-0155.

[51] Grinstein F.F. \& Kailasanath K.K; 1994, "Three Dimensional Numerical Simulations of Unsteady Reactive Square Jets", Comb. \& Flame, 100, p 2.

[52] Westbrook C. \& Dryer F.; 1981, "Simplified Reaction Mechanisms for the Oxidation of Hydrocarbon Fuels in Flames”, Comb. Sci. \& Tech. 27, p 31.

[53] Duwig C. \& Fuchs L.; 2005, "Study of Flame Stabilization in a Swirling Combustor Using a New Flamelet Formulation", Combust. Sci. Tech. 177, 1485.

[54] Fureby C.; 2004, "A Fractal Flame Wrinkling Large Eddy Simulation Model for Premixed Turbulent Combustion”, Proc. Comb, Inst, 30, p 593.

[55] Meneveau C. \& Poinsot T.; 1991, "Stretching and Quenching of Flamelets In Premixed Turbulent Combustion", Comb. \& Flame, 86, p 311.

[56] North G.L. \& Santavicca D.A.; 1990, "The Fractal Nature of Premixed Turbulent Flames", Comb. Sci. \& Tech., 72, p 215. 
[57] Sjunneson A., Olovsson S. \& Sjöblom B.; 1991, "Validation Rig - A Tool for Flame Studies", Presented at the ISABE conference, Nottingham, UK.

[58] Sjunnesson A., Henriksson P. \& Löfström C.; 1992, "CARS Measurements and Visualization of Reacting Flows in a Bluff Body Stabilized Flame" AIAA 92-3650.

[59] Kato H.; 1996, "Cavitation", In Advanced in Marine Hydrodynamics Ed Ohkusu M., Comp. Mech. Publ., Southampton, Boston.

[60] Kubota A., Kato H. \& Yamaguchi H.; 1992, “A New Modeling of Cavitating Flows: A Numerical Study of Unsteady Cavitation on a Hydrofoil Section”, J. Fluid Mech., 240, p 59.

[61] Qin Q., Song C.S. \& Arndt R.E.A.; 2003, "A Virtual Single Phase Natural Cavitation Model and its Application", $5^{\text {th }}$ Int. Symp. on Cavitation, CAV03-OS-1-004.

[62] Wikström N.; 2004, "Modeling Cavitating Flow around a Stationary/Moving Wing Profile", AIAA 04-1287.

[63] Tome M. \& McKee, S.; 1994, "GENSMAC: A Computational Marker and Cell Method for Free Surface Flows in General Domains", J. Comp. Phys., 110, p 171.

[64] Hsiao C. \& Pauli L.; 1998, "Numerical Computation of Tip Vortex Flow Generated by a Marine Propeller", Proceedings of the 1998 ASME Fluids Engineering Division Summer Meeting.

[65] Hirt C. \& Nichols B.; 1981, "Volume Of Fluid (VOF) Method for the Dynamics of Free Boundaries", J. Comp. Phys, 39, p 201.

[66] Ramaprian B. \& Zheng Y.; 1997, "Measurements in Rollup Region of the Tip-Vortex from a Rectangular Wing," AIAA J., 35, p 1263. 